\title{
Significado dos Cuidados Paliativos para a Qualidade da Sobrevivência do Paciente Oncológico
}

\author{
Meaning of Palliative Care for the Quality of Survivorship of Oncologic Patient \\ Significado de los Cuidados Paliativos en la Calidad de la Supervivencia de \\ Paciente Oncológico
}

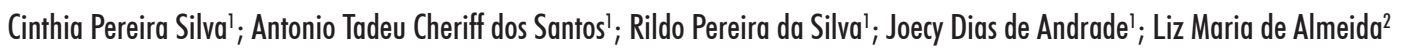

\begin{abstract}
Resumo
Introduçáo: Os avanços e as melhorias no diagnóstico e tratamento do câncer têm proporcionado, no Brasil e no mundo, o aumento do número de sobreviventes. Embora promissor, o aumento de sobreviventes resulta em mais pessoas convivendo com os efeitos crônicos e tardios do câncer e seus tratamentos, requerendo uma nova reflexão acerca dos impactos sociais, da formaçáo de políticas públicas e dos modelos assistenciais voltados para a oferta de cuidados paliativos em oncologia. Objetivo: Compreender as relaçóes de interdependência e complementaridade entre a aplicação do conceito de paliação e qualidade da sobrevivência do paciente oncológico, na perspectiva da equipe multidisciplinar. Método: Pesquisa qualitativa com base na hermenêutica dialética, realizada em hospital de referência em oncologia no Rio de Janeiro, com participação de nove profissionais de saúde integrantes da equipe multiprofissional, com dados produzidos em dois grupos focais, entre julho e agosto de 2015. Resultados: Quatro amplas categorias empíricas foram identificadas: noção de sobrevivência em oncologia; sobrevivência, paliação e linha de cuidados em oncologia; paliação e qualidade da sobrevivência em oncologia; e sobrevivência e avaliação do modelo de paliação institucional. Conclusáo: As necessidades de paliação dos sobreviventes ao câncer demandam políticas específicas de controle de câncer que envolvam a formação de um modelo de planejamento de assistência orientado por uma definição de sobrevivência mais ampliada, fundada em estudos acerca das necessidades físicas, emocionais, econômicas e sociais desse crescente grupo.
\end{abstract}

Palavras-chave: Sobrevivência; Qualidade de Vida; Cuidados Paliativos; Neoplasias; Pesquisa Qualitativa.

\footnotetext{
${ }^{1}$ Núcleo de Pesquisa e Estudos Qualitativos do Instituto Nacional de Câncer José Alencar Gomes da Silva. Ministério da Saúde (NUPEQuali/INCA/ MS). Rio de Janeiro (RJ), Brasil. E-mails: cinthiapereira22@hotmail.com; cheriff@inca.gov.br; rildo@inca.gov.br; jandrade@inca.gov.br ${ }^{2}$ INCA/MS. Rio de Janeiro (RJ), Brasil. E-mail: lalmeida@inca.gov.br Endereço para correspondência: Antonio Tadeu Cheriff dos Santos. Rua Marquês de Pombal, no 125, 7o andar - Centro. Rio de Janeiro. RJ. CEP: 20.230240.E-mail: cheriff@inca.gov.br
} 


\section{INTRODUÇÃO}

De acordo com estimativas do projeto GLOBOCAN $2012^{1}$, houve, em todo o mundo, em 2012, 14,1 milhóes de casos novos de câncer. No Brasil, a estimativa para o ano de 2016 é de aproximadamente de 596 mil novos casos ${ }^{2}$.

Em razão desses indicadores e graças aos avanços e às melhorias no diagnóstico e tratamento precoce ${ }^{3}$, o número de sobreviventes de câncer no mundo tem aumentado. Projeçóes de 2012 estimavam 22,4 milhóes de sobreviventes no mundo ${ }^{4}$, sendo que, só nos Estados Unidos, há a estimativa de que, em 2024, esse número chegará a mais de 19 milhóes de sobreviventes ${ }^{3}$. No Brasil, dados de 2014 também indicam aumento nas taxas de sobrevida em cinco anos para os cânceres de mama e próstata $^{5}$. Embora tais projeçôes sejam promissoras, elas implicam que mais pessoas conviverão com os efeitos crônicos e tardios do câncer e seus tratamentos ${ }^{6}$.

Nesse contexto, o cuidado paliativo se apresenta como uma abordagem especializada passível de dar suporte ao paciente e a seus familiares. Segundo a Organização Mundial da Saúde $(\mathrm{OMS})^{7}$, essa modalidade de cuidado deve ser oferecida o mais precoce possível, a fim de prevenir sintomas e complicaçôes inerentes à doença de base, podendo contribuir para o aumento da qualidade de vida e da sobrevivência do paciente. Para tanto, preconiza como princípios de atuaçáo: o alívio do sofrimento, o controle dos sintomas e da dor, a busca pela autonomia do paciente e a manutenção de vida ativa dos indivíduos enquanto ela durar.

Temel et al. ${ }^{8}$ comentam que os cuidados paliativos têm sido tradicionalmente usados quando o curso avançado da doença ultrapassa as possibilidades curativas disponíveis. Entretanto, os autores apontam que o início tardio da paliação é insuficiente para melhorar a qualidade da vida. Para obter efeito significativo, o cuidado paliativo deve ser fornecido na ocasião do diagnóstico.

Considerando que os princípios do cuidado paliativo comportam uma filosofia de ação que privilegia a promoção da qualidade vida, a prevençáo e o alívio do sofrimento, a discussão da integração precoce da paliação nos cuidados aos sobreviventes de câncer é de extrema importância ${ }^{9}$, uma vez que reforça a importância de uma abordagem multidimensional (física, funcional, emocional, econômica e social) das fases que se seguem ao diagnóstico, tratamento e pós-tratamento do câncer ${ }^{10}$.

Ser um sobrevivente significa viver até o final da vida apesar e para além do câncer, incluindo também os efeitos colaterais e as sequelas decorrentes das terapêuticas utilizadas ${ }^{11}$. O seu controle nâo deve ser considerado uma continuação dos problemas experimentados durante o tratamento, mas um processo e uma linha de cuidado que, para mais das questóes biomédicas, deve ser dinamicamente acompanhado e atendido, buscando a qualidade de vida ${ }^{4,11}$.

No entanto, mais que os aspectos instrumentais e operacionais da aplicação de planos de cuidados e açóes políticas e institucionais voltadas às necessidades dos sobreviventes, a pesquisa procurou interpretar os vários discursos, evidenciando particularidades, confluências e distinçóes acerca da complexidade da sobrevivência, evocadas em meio ao campo de atuação, às relaçóes sociais e aos significados e sentidos atribuídos pelos profissionais de saúde às suas experiências no cuidado aos pacientes oncológicos em paliação ${ }^{12}$.

Assim, o presente estudo objetiva, na perspectiva da equipe multidisciplinar, compreender as relaçóes de interdependência e complementaridade entre a aplicação do conceito de paliaçáo e qualidade da sobrevivência do paciente oncológico.

\section{MÉTODO}

Trata-se de pesquisa qualitativa, fundamentada no enfoque hermenêutico dialético, o qual objetivou a interpretação, compreensão e discussão dos significados contidos nas falas das participantes.

A pesquisa de campo, realizada entre julho e agosto de 2015, em um hospital de referência em Oncologia e Paliação no Rio de Janeiro, logrou aprovação do Comitê de Ética em Pesquisa do Instituto Nacional de Câncer José Alencar Gomes da Silva (CAE 44865115.6.0000.5274em 25/5/2015).

Os dados foram produzidos, nos meses de julho e agosto de 2015, por meio de dois grupos focais audiogravados (duas equipes distintas de profissionais), mediante assinatura do Termo de Consentimento Livre e Esclarecido (TCLE), totalizando nove profissionais de saúde da equipe multiprofissional, atuantes há, pelo menos, um ano em cuidados paliativos na instituição. $\mathrm{O}$ critério de formação dos grupos focais foi a multidisciplinaridade que caracteriza as equipes (enfermagem, fisioterapia, medicina, nutrição, psicologia e serviço social), garantindo a participação de, no mínimo, um profissional de cada categoria, constituindo-se de um assistente social (EA1), uma enfermeira (EE1), dois fisioterapeutas (EF1; EF2), uma médica (EM2), dois nutricionistas (EN1; EN2); duas psicólogas (EP1; EP2). As representaçôes dos fragmentos das falas oriundas das transcriçôes seguem a seguinte notação: $\mathrm{E}$ (entrevistado), seguida da inicial do nome da categoria profissional (A, E, F, M, N, P); e da numeração arábica, de acordo com a ordem cronológica de realização dos dois grupos focais.

Considerando que o grupo focal é uma técnica importante por tratar as questóes sob o ângulo do social, 
estudando as representaçôes e as relaçôes dos diferentes grupos de profissionais da área, o objetivo principal da técnica, no estudo, foi estimular os participantes a debaterem abertamente sobre a relação entre sobrevivência e paliaçáo ${ }^{12}$, quando, mediante roteiro semiestruturado, os participantes foram questionados sobre: definição do que seria a sobrevivência ao câncer e que tipos de pacientes ela abrangeria; momento de indicação e importância dos cuidados paliativos para a qualidade de vida e sobrevivência do paciente oncológico em geral, com câncer avançado ou em terminalidade; avaliação do modelo, da assistência e da estrutura dos cuidados paliativos prestados, considerando os desafios demandados pela perspectiva do aumento do número de sobreviventes. Foram levantados também dados sociodemográficos referentes aos profissionais de saúde (idade; sexo; formação; e tempo de trabalho com cuidados paliativos).

As audiogravaçôes foram transcritas, permitindo a leitura compreensiva do material, facilitando o processo de extração de categorias empíricas, análise, interpretação e identificaçáo de sentidos e o posterior cotejamento entre empiria e teoria. O conjunto dos dados, obtidos por uma amostragem de conveniência, foi fechado pelo princípio de saturação, pela observação da convergência dos discursos e repetição de seus sentidos. $\mathrm{O}$ procedimento metodológico de tratamento dos dados obtidos envolveu: (a) leitura compreensiva; (b) identificação dos sentidos subjacentes às falas dos participantes; (c) problematização das ideias e dos sentidos presentes nos depoimentos e articulação com significados socioculturais; (d) elaboração de síntese entre os dados empíricos e as informaçóes provenientes de outros estudos acerca do assunto, oferecendo aportes teóricos; (e) elaboração de esquemas do processo de análise interpretativa, contendo categorias empíricas e suas correlaçôes com categorias teóricas, gerando sínteses de sentidos ${ }^{12,13}$.

Os referenciais teóricos de análise eleitos foram a hermenêutica da saúde de Gadamer ${ }^{14}$, como reflexão sobre os elementos subjacentes à complexidade da saúde; a noçáo de sobrevivência de Mullan ${ }^{10}$, como precursora da ruptura com a noção biomédica de sobrevivência; e noção de paliação proposta pela $\mathrm{OMS}^{7}$, entendida como abordagem integral em toda a linha de cuidado. Considerando que a experiência da sobrevivência ao câncer é complexa, pressupóe-se que a conjugaçáo desses três arcabouços teóricos melhor contribuiria para a elucidação do fenômeno.

\section{RESULTADOS}

Os participantes eram profissionais de saúde, com idades entre 34 e 49 anos, todos do sexo feminino, com tempo de experiência em oncologia de quatro a 14 anos e em cuidados paliativos entre um e dez anos. Do grupo de participantes, cinco deles atuam exclusivamente em atividades assistenciais e quatro combinavam atividades assistenciais e de gestão.

Considerando os objetivos do estudo, selecionaram-se 28 recortes de falas, que deram origem a quatro amplas categorias empíricas: noçâo de sobrevivência em oncologia; sobrevivência, paliação, e linha de cuidados em oncologia; paliação e qualidade da sobrevivência em oncologia; e sobrevivência e avaliação do modelo de paliação institucional. No interior de cada uma delas, os seus respectivos recortes de falas foram reagrupados em subcategorias empíricas segundo as convergências de significados.

$\mathrm{Na}$ categoria noção de sobrevivência em oncologia, concentra-se o conjunto de concepçôes acerca do que caracteriza e envolve a fase de sobrevivência ao câncer. As caracterizaçóes e significaçóes das subcategorias, a partir das falas que as implicam, são descritas no Quadro 1.

Na categoria sobrevivência, paliação, e linha de cuidados em oncologia agrupa-se o conjunto de falas que indicam o momento da implantaçáo e implementação do modelo de paliação a ser dispensado aos sobreviventes de câncer. As caracterizaçôes e significaçóes das suas subcategorias, a partir das falas que lhes dão origem, são apresentadas no Quadro 2.

A categoria paliação e qualidade da sobrevivência em oncologia abrange o conjunto de falas que indicam as açôes paliativas e suas implicaçóes na qualidade da sobrevivência dos pacientes oncológicos. As caracterizaçóes e significaçôes das suas subcategorias, juntamente com as falas que as caracterizam, são demonstradas no Quadro 3.

A categoria sobrevivência e avaliação do modelo de paliação institucional reúne o conjunto de falas que traduzem as avaliaçóes acerca do modelo de paliação e sua aplicação às necessidades dos sobreviventes ao câncer. As caracterizaçôes e significaçôes das suas subcategorias, a partir das falas que as delineiam, estão ilustradas no Quadro 4.

\section{DISCUSSÃO}

Quatro abrangentes sentidos sociais ganham relevo no material empírico, cada um deles vinculados a uma das categorias e às suas respectivas subcategorias.

\section{O SENTIDO DE COMPLEXIDADE, CONTROVÉRSIAS E POLISSEMIA QUE CONSTITUI A ABORDAGEM E DEFINIÇ̃̃O DA NOÇÃO DE SOBREVIVÊNCIA AO CÂNCER}

Em sua totalidade, as falas constituintes da categoria noção de sobrevivência em oncologia apontam um sentido de complexidade, controvérsia e polissemia acerca da abordagem, caracterização e definição do que consistiria na fase de sobrevivência ao câncer. 
Quadro 1. Depoimentos e atribuição de significados das subcategorias da categoria noção de sobrevivência em oncologia

\begin{tabular}{|c|c|c|}
\hline $\begin{array}{l}\text { Categorias } \\
\text { empíricas }\end{array}$ & Trechos dos depoimentos & Subcategorias empíricas \\
\hline \multirow{5}{*}{ 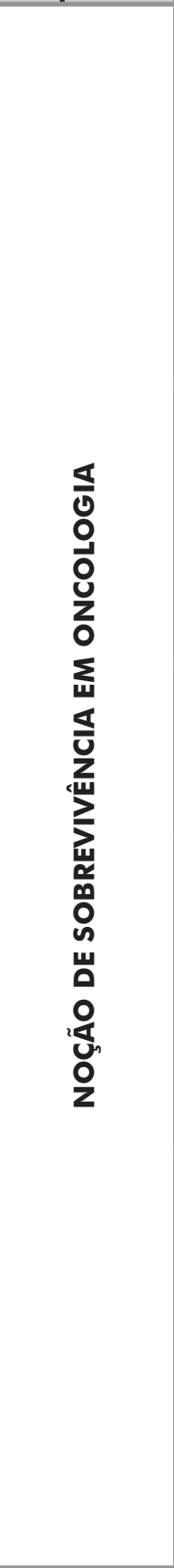 } & $\begin{array}{l}\text { EP2: "Sobrevivente ao câncer [é] todo paciente que } \\
\text { iá passou pelo tratamento curativo e que está em } \\
\text { acompanhamento" } \\
\text { EN2: "Eu entendo a sobrevivência como alguém que } \\
\text { passou por todo o tratamento e tem um [prognóstico] } \\
\text { de cura" } \\
\text { EM2: "A minha concepção seria (...) um paciente com } \\
\text { diagnóstico de câncer [que] passou por um tratamento } \\
\text { e, depois de um tempo já teve o critério de cura" }\end{array}$ & $\begin{array}{l}\text { Sobrevivência entendida a partir } \\
\text { do modelo biomédico oncológico } \\
\text { tradicional } \\
\text { Conjunto de concepções de sobrevivência } \\
\text { como um processo mediado pelos } \\
\text { parâmetros biomédicos temporais de } \\
\text { tratamento, remissão e acompanhamento } \\
\text { do caso de câncer }\end{array}$ \\
\hline & $\begin{array}{l}\text { EN2: "O significado de sobrevivência da [traduz] uma } \\
\text { sensação de vencer" } \\
\text { EF1: "(...) sobrevivente realmente [é] aquele que venceu } \\
\text { [doença]" } \\
\text { EA1: "(...) a sobrevivência é um tempo árduo de luta } \\
\text { mesmo e o paciente vem com forças bem escassas" }\end{array}$ & $\begin{array}{l}\text { Sobrevivência entendida como luta e } \\
\text { superação da doença } \\
\text { Conjunto de concepções de sobrevivência } \\
\text { como uma opção de luta pela vida e um } \\
\text { estado de superação e vitória do paciente } \\
\text { oncológico sobre a doença }\end{array}$ \\
\hline & $\begin{array}{l}\text { EN1: "Eu acho que essa sobrevivência começa a partir } \\
\text { do momento do diagnóstico" } \\
\text { EP1: "Ele [o paciente] começa a luta [pela] sobrevivência } \\
\text { desde o diagnóstico da doença oncológica" }\end{array}$ & $\begin{array}{l}\text { Sobrevivência entendida a partir do } \\
\text { diagnóstico } \\
\text { Conjunto de concepções de sobrevivência } \\
\text { como um estado iniciado a partir do } \\
\text { momento do diagnóstico do câncer }\end{array}$ \\
\hline & $\begin{array}{l}\text { EA 1: "sobrevivência (...) é realmente uma luta (...) com } \\
\text { sequelas advindas do tratamento, sequelas advindas } \\
\text { do câncer, e com uma demanda [social] para além do } \\
\text { câncer" } \\
\text { EN 1: "Sobrevivência se estende [para] além do } \\
\text { paciente. Ela afeta todo o entorno dele, a família [e] } \\
\text { toda estruturação social, psicológica (...) eu não consigo } \\
\text { ver a sobrevivência em um foco só" }\end{array}$ & $\begin{array}{l}\text { Sobrevivência entendida como um } \\
\text { processo amplo e complexo } \\
\text { Conjunto de concepções de sobrevivência } \\
\text { como um processo estruturalmente } \\
\text { complexo, determinado dinamicamente } \\
\text { pelos aspectos clínicos da doença e } \\
\text { apoios social e familiar disponíveis ao } \\
\text { paciente }\end{array}$ \\
\hline & $\begin{array}{l}\text { EM2: "Eu entendo (...) [a sobrevivência] a partir do } \\
\text { diagnóstico até o momento que ele está respondendo } \\
\text { a alguma intervenção, oncológica especifica (...)" } \\
\text { EM2: "(...) a partir do momento que ele entra em } \\
\text { cuidado de final de vida ele já não está mais com vida; } \\
\text { ele não sobrevive, ele está morrendo (...)" } \\
\text { EA1: "(...) acredito que se [o paciente] já está em um } \\
\text { processo de finitude mesmo; de cuidados de fim de } \\
\text { vida (...) eu acho que já muda um pouco a categoria } \\
\text { desse paciente" }\end{array}$ & $\begin{array}{l}\text { Sobrevivência entendida como a } \\
\text { existência de respostas do paciente } \\
\text { às intervenções e aos cuidados } \\
\text { Conjunto de concepções de sobrevivência } \\
\text { como um estado determinado pela } \\
\text { existência de respostas do paciente aos } \\
\text { cuidados dispensados }\end{array}$ \\
\hline
\end{tabular}

A sobrevivência ao câncer é uma experiência complexa podendo possuir vários significados e sentidos dependendo do grupo (pacientes, profissionais de saúde e familiares), e tipo de abordagem. A definição de sobrevivente tem sido, desde a década de 1980, objeto de amplos debates, controvérsias e desencontros que ainda envolvem a necessidade de consolidação de uma noção comum de sobrevivência que seja consensualmente aceita e reconhecida ${ }^{15,16}$.

\section{A sobrevivência entendida a partir do modelo biomédico} oncológico tradicional traduz, de forma plena, o sentido da sobrevivência mediado pela lógica do modelo biomédico oncológico tradicional: "A minha concepção seria [...] um paciente com diagnóstico de câncer [que] passou por um tratamento e, depois de um tempo, já teve o critério de cura" (EM2). Caracterizado por uma definição clínica da sobrevivência baseada em parâmetros biomédicos temporais do fim do tratamento, remissão 
Quadro 2. Depoimentos e atribuição de significados das subcategorias da categoria sobrevivência, paliação, e linha de cuidados em oncologia

\begin{tabular}{|c|c|c|}
\hline $\begin{array}{l}\text { Categorias } \\
\text { empíricas }\end{array}$ & Trechos dos depoimentos & Subcategorias empíricas \\
\hline 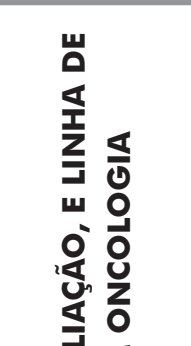 & $\begin{array}{l}\text { EP1: "Eu acho que [é] o momento do diagnóstico (...). } \\
\text { Desde o diagnóstico, [os pacientes] deveriam estar } \\
\text { inseridos [na] esfera dos cuidados paliativos (...). Se no } \\
\text { diagnóstico incluíssemos esse processo, a gente teria } \\
\text { mais efetividade em relação a resposta ao tratamento" } \\
\text { EF1: "Desde o [momento do] diagnóstico, a gente já } \\
\text { [deveria] começar com essa visão (...)" }\end{array}$ & $\begin{array}{l}\text { Paliação na sobrevivência como um } \\
\text { modelo de ação e cuidado iniciado } \\
\text { a partir do diagnóstico e início do } \\
\text { tratamento } \\
\text { Conjunto de falas que indicam que as } \\
\text { ações e cuidados de paliação devem ser } \\
\text { iniciadas a partir da fase do diagnóstico e } \\
\text { tratamento do câncer }\end{array}$ \\
\hline 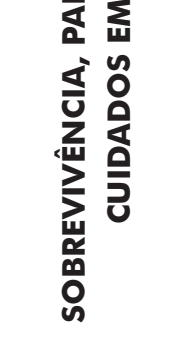 & $\begin{array}{l}\text { EN2: "A divisão cuidado paliativo, [cuidado] curativo é } \\
\text { bem estruturada e fundamentada (...). Essa separação } \\
\text { ainda é muito pertinente em muitos momentos" } \\
\text { EF2: "O melhor seria obviamente desde o início, mas, } \\
\text { como a gente tem essa estrutura, essa definição de tipo } \\
\text { curativo, a paliação [após os cuidados curativos] facilita } \\
\text { muito o nosso trabalho" }\end{array}$ & $\begin{array}{l}\text { Paliação na sobrevivência como um } \\
\text { modelo de ação e cuidado iniciado } \\
\text { a partir do término do tratamento } \\
\text { curativo }\end{array}$ \\
\hline
\end{tabular}

Quadro 3. Depoimentos e significações das subcategorias da categoria paliação e qualidade da sobrevivência em oncologia

\begin{tabular}{|c|c|c|}
\hline $\begin{array}{l}\text { Categorias } \\
\text { empíricas }\end{array}$ & Trechos dos depoimentos & Subcategorias empíricas \\
\hline \multirow{2}{*}{ 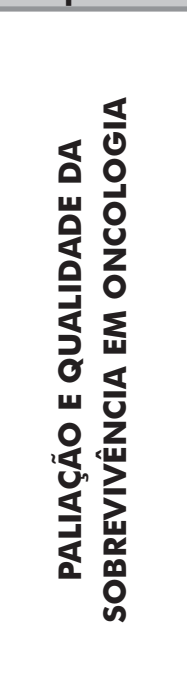 } & $\begin{array}{l}\text { EF2: "A gente tenta manejar os sintomas; dar um } \\
\text { acolhimento para um paciente que chega aqui cansado } \\
\text { e muito descrente } \\
\text { Dentro do possível, conseguimos fazer um trabalho e } \\
\text { dar um pouco de qualidade de vida para ele" } \\
\text { EP2: "os cuidados paliativos são muito importantes } \\
\text { na fase de sobrevivência porque não importa só } \\
\text { sobreviver, o importante é saber como essas pessoas } \\
\text { estão sobrevivendo a essa doença" }\end{array}$ & $\begin{array}{l}\text { Paliação na sobrevivência como ações } \\
\text { de conforto e controle de sintomas } \\
\text { Conjunto de falas que indicam o conforto, } \\
\text { o controle dos sintomas e a mitigação do } \\
\text { sofrimento como um diferencial para a } \\
\text { qualidade da sobrevivência em oncologia }\end{array}$ \\
\hline & $\begin{array}{l}\text { EF2: "É um trabalho que realmente traz qualidade } \\
\text { de vida e a gente espera ao máximo trazer uma boa } \\
\text { qualidade de morte" } \\
\text { EM2: "A gente consegue observar impacto positivo na } \\
\text { vida dos pacientes, na forma como eles morrem, na } \\
\text { forma como eles ficam até o momento da morte e na } \\
\text { questão dos familiares mesmo" }\end{array}$ & $\begin{array}{l}\text { Paliação na sobrevivência como } \\
\text { preparo para uma boa morte } \\
\text { Conjunto de falas que implicam o } \\
\text { preparo para uma boa morte como } \\
\text { um diferencial para a qualidade da } \\
\text { sobrevivência em oncologia }\end{array}$ \\
\hline
\end{tabular}

e/ou acompanhamento do caso de câncer, esse sentido coincide com a definição defendida pela European Organization of Research ${ }^{16}$. Entretanto, essa concepção tem como ponto fraco a exclusão dos pacientes em terapia de manutenção. Ademais, simplifica a sobrevivência como estado binário de cura ou presença da doença ${ }^{16}$. Essa percepção não só oculta o processo de compreensão da saúde $^{14}$ como não captura a experiência de longo prazo da sobrevivência, simplificando um processo vital único de diferentes indivíduos ${ }^{10,14}$.

A sobrevivência entendida como luta e superação da doença envolve discursos que remetam a um sentido de sobrevivência como atitude assertiva do paciente de luta pela vida e superação da doença: "[...] sobrevivente realmente [é] aquele que venceu [doença]” (EF1). Embora esse sentido aponte uma concepção mais aberta de sobrevivência e vise a incentivar e a reforçar um comportamento combativo e esperançoso do paciente, ele continua restrito por uma visão tradicional de sobrevivência associada à cura. Uma visão que guarda relação com o modelo biomédico tradicional, encobrindo o sentido da doença e da saúde ${ }^{14}$, e, por conseguinte, da sobrevivência ${ }^{10}$, ao náo capturar a sua experiência de longo prazo. 
Quadro 4. Depoimentos e significações das subcategorias da categoria Sobrevivência e avaliação do modelo de paliação institucional

\begin{tabular}{|c|c|c|}
\hline $\begin{array}{l}\text { Categorias } \\
\text { empíricas }\end{array}$ & Trechos dos depoimentos & Subcategorias empíricas \\
\hline 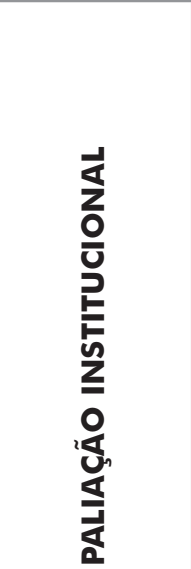 & $\begin{array}{l}\text { EA 1: "Na verdade o cuidado [paliação] tinha que } \\
\text { perpassar tudo [toda a linha de cuidado] desde o inicio } \\
\text { do tratamento, até [a fase] paliativa" } \\
\text { EP2: "No Brasil, a gente ainda atua nos cuidados } \\
\text { paliativos na fase de final de vida. (...) se a gente pensar } \\
\text { em um cuidado paliativo de uma maneira mais ampla, } \\
\text { inserido desde o diagnóstico, seja no caso da oncologia } \\
\text { como de outras doenças crônicas, eu acho que teríamos } \\
\text { um resultado melhor em termos de qualidade da } \\
\text { sobrevivência" } \\
\text { EM2: "O ideal seria que a gente conseguisse integrar } \\
\text { o cuidado paliativo, desde o diagnóstico do paciente } \\
\text { até o momento do final de vida" }\end{array}$ & $\begin{array}{l}\text { Modelo e planos de cuidados como } \\
\text { necessitados de uma política pública } \\
\text { Conjunto de falas que traduzem de } \\
\text { ações políticas necessárias para a } \\
\text { reorganização e integração do modelo } \\
\text { de paliação às necessidades dos } \\
\text { sobreviventes de câncer }\end{array}$ \\
\hline 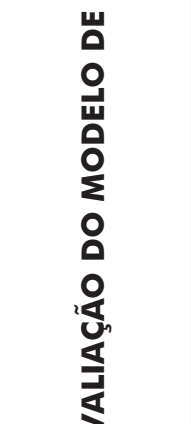 & $\begin{array}{l}\text { EN 1: "A gente iá esbarra na formação desses } \\
\text { profissionais de cuidado paliativo; dificuldade de as } \\
\text { pessoas entenderem o que é fazer cuidados paliativos" } \\
\text { EM2: "O que seria fundamental seria difundir a } \\
\text { educação em cuidados paliativos nas universidades, } \\
\text { melhorar os cursos de capacitação (...). Não há uma } \\
\text { capacitação em cuidados paliativos dos profissionais } \\
\text { das unidades primárias. Infelizmente, os pacientes têm } \\
\text { dores mal controladas durante meses, além de outros } \\
\text { sintomas" }\end{array}$ & 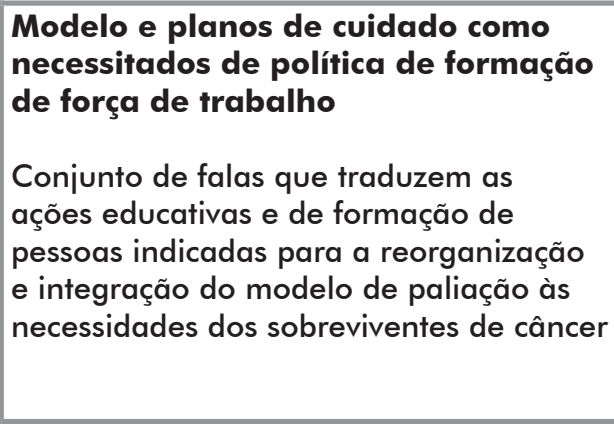 \\
\hline 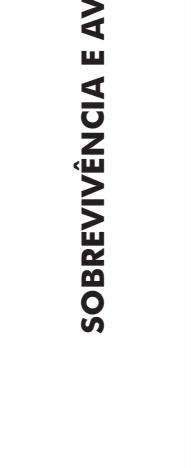 & $\begin{array}{l}\text { EN2: Todo diferencial é o trabalho em equipe. } \\
\text { Esse trabalho integrado no qual os profissionais se } \\
\text { comunicam. Essa questão da troca, do acolhimento. } \\
\text { ser paliativista envolve outras questões, além da mera } \\
\text { técnica individual do nutricionista, do fisioterapeuta, } \\
\text { psicologia. A gente percebe que esse vínculo e essa } \\
\text { comunicação entre [membros da] equipe é uma questão } \\
\text { importante para o cuidado desse paciente". } \\
\text { EM2: "a comunicação é ainda um grande desafio para } \\
\text { a gente, eu acho que outra questão é o prognostico e } \\
\text { o próprio trabalho" }\end{array}$ & $\begin{array}{l}\text { Modelo e planos de cuidados como } \\
\text { necessitados de uma nova forma de } \\
\text { trabalho em saúde } \\
\text { Conjunto de falas que traduzem as } \\
\text { ações de estruturação de equipes e de } \\
\text { planejamento de ações de gestão do } \\
\text { trabalho em saúde indicados para a } \\
\text { reorganização e integração do modelo } \\
\text { de paliação às necessidades dos } \\
\text { sobreviventes de câncer }\end{array}$ \\
\hline
\end{tabular}

A sobrevivência entendida a partir do diagnóstico é compreendida como experiência iniciada a partir do momento da alteraçáo do estado de saúde de uma pessoa e determinada pela confirmação diagnóstica do câncer: "Ele [o paciente] começa a luta [pela] sobrevivência desde o diagnóstico da doença oncológica" (EP1). Apesar de esse sentido de sobrevivência ainda ser mediado por um marco temporal e uma intervenção clínico-diagnóstica, ele traz uma ampliação da percepçáo da sobrevivência para além da tradicional perspectiva biomédica. A proposta de Mullan ${ }^{10}$, na qual os conceitos de curado e doente não são suficientes para descrever as implicaçóes emocionais, físicas e sociais do acometimento pelo câncer, é reforçada pela US National Coalition of Cancer Survivors ${ }^{17}$. Nesse caso, a sobrevivência é deflagrada no momento do diagnóstico, estendendo-se por toda a vida do paciente $e^{9,16,17}$.
Essa abordagem visa a transformar o tradicional papel de vitimado do paciente em outro mais proativo e esperançoso ${ }^{11}$. Embora essa definição enfatize uma gama de problemas e necessidades dos pacientes com câncer, sua característica é mais política (advocacy) do que científica ${ }^{16}$, demandando pesquisas sobre as reais necessidades dos sobreviventes decorrentes dos diversos tipos de câncer e da sua real condição e autoaceitação como sobrevivente ${ }^{15}$.

A sobrevivência entendida como um processo amplo e complexo apresenta a experiência da sobrevivência mediada pelo processo vivencial e clínico do paciente e suas relaçôes com as estruturas familiares, socioeconômicas e sociotécnicas da saúde: "Sobrevivência se estende [para] além do paciente. Ela afeta todo o entorno dele, a família, toda estruturação social, psicológica. [...] eu não consigo ver a sobrevivência em um foco só" (EN1). 
Nesse caso, a definição de sobrevivência não só busca ir além das restriçóes de tempo e tipo de tratamento, abrangendo diversas experiências e trajetórias individuais e familiares ${ }^{11}$, como também envolve relaçóes com as estruturas econômicas e técnicas da área da saúde, descortinadas pelos casos e pelas demandas operacionais das diversas condiçóes do adoecimento: pacientes livres de doença ou com doença ativa; em recidiva ou com períodos intermitentes da doença; em novos tratamentos; com um segundo tipo de câncer; ou com complicaçôes tardias da doença ou do tratamento ${ }^{18}$.

Para a atual conjuntura do debate envolvendo as necessidades dos sobreviventes ao câncer, não existe consenso sobre quando começa a sobrevivência; se é após o tratamento, após a fase de cinco anos de pós-diagnóstico com câncer avançado, ou separado do cuidado de fim de vida ${ }^{15,16}$.

A sobrevivência entendida como a existência de respostas do paciente às intervençóes e cuidados evidencia a sobrevivência mediada pelo grau de resposta às intervençôes e aos cuidados biomédicos. A questâo a ser discutida aqui é o sentido atribuído a essa intervenção específica. Teria ela um sentido compreensivo, abarcando uma noção mais ampla da paliação? "Eu entendo [...] [a sobrevivência] a partir do diagnóstico até o momento que ele está respondendo a alguma intervenção, ainda que não seja uma intervençâo oncológica específica [...]” (EM2); ou tenderá a uma noção fragmentada do próprio cuidado paliativo? "A partir do momento que ele entra em cuidado de final de vida, ele já não está mais com vida; ele não sobrevive, ele está morrendo" (EM2). A resposta é que esse sentido, subsidiário do modelo biomédico, ilumina a noção de sobrevivência e da aplicação de cuidados a pacientes com câncer avançado e em cuidados de fim de vida.

Se o desvelamento do sentido permite entrever uma compreensão que não se pauta apenas na intervenção oncológica, tendo em vista outra perspectiva ilustrada por fala, cabe indagar se o indivíduo em processo de morte é alguém que ainda sobrevive. Se não, o que ele é? Vejamos o que responde o depoente: "Acredito que se [o paciente] já está em um processo de finitude mesmo; de cuidados de fim de vida [...] eu acho que já muda um pouco a categoria desse paciente" (EA1).

Em um sentido ético-filosófico, Gadamer ${ }^{14}$ argumenta que, se uma pessoa excluir de si e do viver a ideia da morte, é como se ela estivesse excluindo-se a si própria. Infere-se que pode haver nas falas uma estratégia defensiva da equipe, na busca de evitar o confronto com a iminência da morte; morte interdita ${ }^{19}$. Ocorreria, nesse caso, uma morte social antecipada ${ }^{19}$. São concepçóes que acabariam não só por náo considerar a trajetória de vida do sujeito em terminalidade ${ }^{15}$ como sobrevivente, como fragilizariam a própria equipe ${ }^{19}$. Nada mais distante da combinação entre as atuais noçóes de sobrevivência e paliação.

Os cuidados paliativos não devem ser destinados apenas aos pacientes no pós-tratamento curativo, devendo ser considerados em toda a linha de cuidados, independente do fim de vida ${ }^{20}$. A discussão que se abre acerca da natureza desse cuidado paliativo é de não cair na excessiva instrumentalização e especialização do cuidado $^{14,21}$, determinadas pela lógica da linha de ação do tradicional modelo biomédico oncológico.

\section{O SENTIDO DA CONVENIÊNCIA E OPORTUNIDADE DA IMPLANTAÇÃO DO MODELO DE PALIAÇÃO INTEGRADO À LINHA DE CUIDADOS DOS SOBREVIVENTES DE CÂNCER}

As falas da categoria sobrevivência, paliação e linha de cuidados em oncologia evidenciam a conveniência e a oportunidade da implantação e implementação do modelo de paliação integrado à linha de cuidados aos sobreviventes de câncer. A definição de sobrevivente do National Coalition for Cancer Survivorship (NCCS) ${ }^{17}$ incorporando os "que estáo passando por tratamento primário; aqueles que estấo em remissáo e no pós-tratamento; aqueles que estâo curados e também aqueles que estão com doença ativa ou doença avançada" ${ }^{15}$ abre um espaço de inclusão dos pacientes em fim de vida ${ }^{7}$, apesar de historicamente não serem abrangidos pela definição tradicional de sobrevivente.

A paliação na sobrevivência como um modelo de ação e cuidado iniciado a partir do diagnóstico e início do tratamento refere-se à caracterização da orientação do cuidado na sobrevivência ao câncer como uma proposta de paliação antecipada:

Eu acho que [é] o momento do diagnóstico [...]. Desde o diagnóstico [os pacientes] deveriam estar inseridos [na] esfera dos cuidados paliativos [...]. Se no diagnóstico incluíssemos esse processo, a gente teria mais efetividade em relação a resposta ao tratamento (EP1).

Diversos autores ${ }^{9,20,22,23}$ apontam para a necessidade da formação de um modelo integrado de paliação desde o início do tratamento do paciente com câncer, no qual a aplicação do cuidado paliativo, de forma individualizada e adaptada à evolução da doença, seja associada ao cuidado curativo. Tudo isso com a finalidade de melhoria e racionalização da assistência; redução dos custos assistenciais e promoção da qualidade de vida, seja por diminuição das internaçôes por quadros agudos ou casos de emergência. Essas medidas - valores padronizados -, no entanto, não devem se sobrepor ou substituir a necessidade também de um cuidado humanizado ${ }^{14}$. 
A paliação na sobrevivência como um modelo de ação e cuidado iniciado a partir do término do tratamento curativo caracteriza o modelo de cuidado na sobrevivência ao câncer como uma açáo após o tratamento biomédico curativo: "A divisão cuidado paliativo, [cuidado] curativo é bem estruturada e fundamentada [...]. Essa separação ainda é muito pertinente em muitos momentos" (EN2). Temel et al. ${ }^{8}$ apontam que, apesar de os cuidados paliativos serem tradicionalmente oferecidos após o término do tratamento curativo, esse tipo de cuidado é insuficiente para alterar a qualidade de vida dos pacientes. As recomendaçóes de Economou', Parikh et al. ${ }^{20}$, Bruera e Yennurajalingam ${ }^{22} \mathrm{e}$ Temel et al. ${ }^{8}$ são coincidentes em dois pontos: na descrição da diferença que a gestáo de cuidados paliativos traz para a melhoria do cuidado aos pacientes oncológicos com doença avançada e no final de vida; e na descrição de como as intervençôes de cuidados paliativos incrementam a qualidade de vida dos pacientes em qualquer idade ou fase de doença, inclusive para aqueles cujas terapêuticas foram esgotadas ou se encontram em cuidados de fim de vida.

A justificativa para esses últimos também serem considerados dentro de uma mesma linha de cuidado, apesar da sua dinâmica e peculiaridade, reside no sentido maior de a paliação e a própria sobrevivência serem contempladas pelos sistemas de saúde como processos individualizados.

\section{O SENTIDO DA PALIAÇÃO COMO UMA DIRETRIZ ESSENCIAL PARA A QUALIDADE DA SOBREVIVÊNCIA DO PACIENTE ONCOLÓGICO}

Os depoimentos da categoria paliação e qualidade da sobrevivência em oncologia evidenciam a paliação como uma diretriz essencial para a qualidade da sobrevivência do paciente oncológico, tanto por meio de açóes de conforto e controle de sintomas como de preparo para uma boa morte ${ }^{21}$.

A paliação na sobrevivência como açóes de conforto e controle de sintomas traduz a caracterizaçáo do modelo de cuidado na sobrevivência ao câncer como um processo de controle de sintomas e mitigação do sofrimento:

A gente tenta manejar os sintomas; dar um acolhimento para um paciente que chega aqui cansado e muito descrente. Dentro do possível, conseguimos fazer um trabalho e dar um pouco de qualidade de vida para ele (EF2).

Segundo a $\mathrm{OMS}^{7}$, o cuidado paliativo, por meio da prevenção e tratamento da dor e de outros problemas de natureza espiritual, psicossocial e física, atua como uma abordagem promotora da qualidade de vida dos pacientes e familiares.
No entanto, apesar de a avaliação da qualidade de vida dos sobreviventes ser importante para a identificação da condição global da vida do paciente e para a verificação do serviço prestado ${ }^{23}$, não se deve sobrepô-la à humanização. Revisitando Gadamer ${ }^{14}$, as medidas e açōes na área da saúde não podem se orientar apenas por valores padrôes. Devem privilegiar o olhar, o ouvir e o sentir que acolham o paciente como pessoa.

A paliação na sobrevivência como preparo para uma boa morte abarca discursos que traduzem o modelo de cuidado na sobrevivência ao câncer como uma ação biomédica direcionada para o fim de vida do paciente:

A gente consegue observar impacto positivo na vida dos pacientes, na forma como eles morrem, na forma como eles ficam até o momento da morte e na questão dos familiares mesmo (EM2).

Assim, o cuidado paliativo tem, além da possibilidade de resgatar a morte como evento natural e esperado ${ }^{23}$, priorizar a vida, promovendo a dignidade do indivíduo e mitigando o sofrimento.

$\mathrm{Na}$ linha das reflexóes de Gadamer ${ }^{14}$ acerca da natureza $e$ da arte da medicina e sobre o caráter oculto da saúde, a paliação, como uma técnica, projeto de especialidade médica ${ }^{21}$, mais que evocar um tratamento mágico de eliminação da doença ou de medicalização da morte, deve ser a humanização da mais crônica de todas as doenças: a perspectiva de morte que acompanha todo ser humano. Para Gadamer, a medicina aprendeu a tratar de muitas doenças. Entretanto, não é capaz de erradicá-las. $\mathrm{Na}$ impossibilidade de eliminação de todas as doenças ou, mesmo da negaçáo da morte, nos restaria aprender a aceitá-las e saber encontrar o nosso mais certo destino: no caso, cuidar do sobrevivente na sua condiçáo crônica, de terminalidade e/ou de fim de vida. Cuidar não apenas de um caso ou de uma entidade mórbida, mas de um indivíduo que tem na sua morte a realização da sua humana possibilidade final. A paliaçáo, nesse sentido, mais que do domínio técnico do morrer, se abre na difícil tarefa que é a de aprender a aceitar o limitado, o dolorido; ouvindo, vendo e dialogando com a "vida" na sua finitude.

\section{O SENTIDO DE NECESSIDADE DE ORGANIZAÇÃO DO MODELO ASSISTENCIAL DE PALIAÇÃO PARA O PLANEJAMENTO E ORDENAÇÃO DOS PLANOS DE CUIDADOS PARA OS SOBREVIVENTES DE CÂNCER}

As falas da categoria sobrevivência e avaliação do modelo de paliação institucional indicam a necessidade de organização do modelo assistencial de paliação para o planejamento e a ordenaçáo dos planos de cuidados para os sobreviventes ao câncer, tanto para a formação de 
uma política pública nacional de paliação, como para a qualificação profissional e de uma nova forma de trabalho em saúde para além do modelo biomédico.

Em modelo e planos de cuidados como necessitados de uma política pública, os discursos apontam a urgência da incorporação da paliação a toda linha de cuidado, legitimada na referida política pública nacional de paliação: "O ideal seria que a gente conseguisse integrar o cuidado paliativo, desde o diagnóstico do paciente até o momento do final de vida" (EM2). Vários autores ${ }^{20,22,24}$ apontam a exigência da formação de um modelo integrado de paliação desde o início do tratamento do paciente com câncer, no qual o cuidado paliativo seja concebido e aplicado em associação com o cuidado curativo. Floriani e Schramm ${ }^{25}$ igualmente salientam a importância da implantação dos cuidados paliativos no âmbito do sistema de saúde brasileiro, indicando que este ainda sofre da falta de articulação entre os diferentes níveis assistenciais.

Já as falas da subcategoria modelo e planos de cuidados como necessitados de política de formação de força de trabalho indicam a necessidade da constituição de uma política de formação profissional que, além de vincular-se à mencionada política pública nacional de paliação, seja capaz de atender quantitativa e qualitativamente às demandas dos cuidados paliativos:

$\mathrm{O}$ que seria fundamental seria difundir a educação em cuidados paliativos nas universidades, melhorar os cursos de capacitação [...]. Não há uma capacitaçáo em cuidados paliativos dos profissionais das unidades primárias. Infelizmente, os pacientes têm dores mal controladas durante meses, além de outros sintomas (EM2).

Nessa abordagem, o cuidado será também o de não cair em um modelo de especialização técnica, mas sim um modelo que "ajude a ressignificaçâo social do sofrimento, da dor, da doença e da morte, a partir de quem as vive" 14 .

O modelo e plano de cuidados como necessitados de uma nova forma de trabalho em saúde remete ao sentido de estruturação de programas e planos de cuidados de paliação na sobrevivência baseados no trabalho em equipe em uma abordagem interdisciplinar do sobrevivente:

Todo diferencial é o trabalho em equipe. Esse trabalho integrado no qual os profissionais se comunicam. Essa questão da troca, do acolhimento. O ser paliativista envolve outras questôes, além da mera técnica individual do nutricionista, do fisioterapeuta, psicologia. A gente percebe que esse vínculo e essa comunicaçáo entre [membros da] equipe é uma questão importante para o cuidado desse paciente (EN2).
Para que os cuidados paliativos com qualidade estejam disponíveis para os sobreviventes, será necessário mais que a execução de uma técnica perfeita; ou seja, mais que um ser-capaz-de-fazer-seguro, uma techne ${ }^{14}$ relacionada à antecipação e gestão dos sintomas decorrentes do câncer ou do seu tratamento, integrada a um "ser-capaz-de-fazerorientado" 14 , na qual a trajetória do sobrevivente seja interpretada, avaliada e reelaborada hermeneuticamente, privilegiando a preocupação com o outro que sofre, vive e morre $^{14}$. Uma das condiçôes para o êxito desse intento é a comunicação efetiva e eficaz entre equipe multidisciplinar, pacientes e cuidadores ${ }^{9,23}$.

\section{CONCLUSÃO}

Considerado o conjunto dos significados e sentidos em torno do objeto investigado, na linha de um modelo de advocacy e de um tradicional cuidado oncológico, o estudo vislumbra os diversos conceitos enunciados pelo atual debate acerca da sobrevivência ao câncer. Apesar de oriundos do modelo biomédico, tais sentidos e significados conformam uma expectativa que transcende os seus limites, apontando para além dele, instaurando uma busca que, ainda ao seu modo técnico, tenta resgatar a dignidade do paciente e a significação existencial da sua trajetória social e individual.

No que diz respeito à aplicaçấo dos cuidados paliativos para a qualidade da sobrevivência do paciente oncológico, os sentidos voltam-se para a necessidade de adequaçáo das atuais linhas de cuidado, pois as consequências da doença e do seu tratamento, entendidos como exigências para um plano operacional de cuidado e um resgate social da trajetória desse paciente, constituem os desafios a serem enfrentados.

Ante os achados do estudo, as recomendações possíveis apontam para a necessidade de um modelo de atenção em que a aplicação dos cuidados paliativos não seja fragmentada, mas proporcionada de forma humanizada e integrada desde o diagnóstico, atingindo todo o continuum das intervençôes terapêuticas/curativas, sem negligenciar o fim de vida, quando todo processo da sobrevivência encontra seu termo.

Particularmente, quanto às necessidades de paliação dos sobreviventes ao câncer, os desafios demandados envolvem políticas específicas de controle de câncer, preconizando a formação de um modelo de planejamento de assistência em paliação orientado por uma definição de sobrevivência mais ampliada, fundada em estudos acerca das necessidades físicas, emocionais, econômicas e sociais desse grupo que é crescente no mundo.

\section{CONTRIBUIÇÕES}

Cinthia Pereira Silva participou da concepção, delineamento, análise, interpretação dos dados e redação 
do artigo. Antonio Tadeu Cheriff dos Santos orientou e colaborou na concepção, delineamento, análise, interpretação dos dados, redação do artigo e aprovação da versão a ser publicada. Rildo Pereira da Silva, Joecy Dias de Andrade e Liz Maria de Almeida colaboraram na análise, interpretação dos dados, redação do artigo, revisão crítica e aprovaçáo da versão a ser publicada.

\section{Declaração de Conflito de Interesses: Nada a Declarar.}

\section{REFERÊNCIAS}

1. Ferlay J, Soerjomataram I, Ervik M, Dikshit R, Eser S, Mathers C, et al. GLOBOCAN 2012: cancer incidence and mortality worldwide. Lyon: International Agency for Research on Cancer; 2013. (IARC CancerBase, 11).

2. Instituto Nacional de Câncer José de Alencar Gomes da Silva. Estimativa 2015: incidência de câncer no Brasil. Rio de Janeiro: Inca; 2015.

3. Desantis CE, Lin CC, Mariotto AB, Siegel RL, Stein $\mathrm{KD}$, Kramer JL, et al. Cancer treatment and survivorship statistics, 2014. CA Cancer J Clin. 2014;64 (4):252-71.

4. Reuben SH. Living beyond cancer: finding a new balance. Washington, D.C.: U.S. Department of Health and Human Services; 2004 may. 2003-2004 annual report.

5. Allemani C, Weir HK, Carreira H, Harewood R, Spika D, Wang XS, et al. Global surveillance of cancer survival 1995-2009: analysis of individual data for 25676887 patients from 279 population-based registries in 67 countries (CONCORD-2). Lancet. 2015;385(9972):977-10106.

6. Alfano CM, Molfino A, Muscaritoli M. Interventions to promote energy balance and cancer survivorship: priorities for research and care. Cancer. 2013 Jun 1;119 Suppl 11:2143-50.

7. World Health Organization. Paliative Care: fact sheet $\mathrm{n}^{\circ}$ 402 [Internet]. Geneva: WHO; 2015 [acesso 2016 feb 20]. Disponível em: http://www.who.int/mediacentre/ factsheets/fs402/en/.

8. Temel JS, Greer JA, Muzikansky A, Gallagher ER, Admane S, Jackson VA, et al. Early palliative care for patients with metastatic non-small-cell lung cancer. $\mathrm{N}$ Engl J Med. 2010;363(8):733-42.

9. Economou D. Palliative care needs of cancer survivors. Semin Oncol Nurs. 2014;30(4):262-7.
10. Mullan F. Seasons for survival: reflections of a physician with cancer. N Engl J Med. 1985;313(4):270-3.

11. Muniz RM, Zago MMF, Schwartz E. As teias da sobrevivência oncológica: com a vida de novo. Texto Contexto-Enferm. 2009;18(1):25-32.

12. Minayo MCS. O desafio do conhecimento: pesquisa qualitativa em saúde. São Paulo: Hucitec; 2008.

13. Gomes R. Análise e interpretação de dados de pesquisa qualitativa. In: Minayo MCS., organizadora. Pesquisa social: teoria, método e criatividade. Petrópolis: Vozes; 2007. p. 79-108.

14. Gadamer HG. O caráter oculto da saúde. 2 ed. Petrópolis: Vozes; 2011.

15. Khan NF, Rose PW, Evans J. Defining cancer survivorship: a more transparent approach is needed. J Cancer Surviv. 2012;6(1):33-6.

16. Dirven L, Van de Poll-Franse LV, Aaronson NK, Reijneveld JC. Controversies in defining cancer survivorship. Lancet Oncol. 2015;16(6):610-2.

17. National Coalition for Cancer Survivorship. NCCS Mission [Internet]. Silver Spring: NCCS; [acesso em 2016 jan 21]. Disponível em: http://www.canceradvocacy.org/ about-us/our-mission/.

18. American Cancer Society. Cancer treatment and survivorship facts \& figures: 2012-2013. Atlanta: American Cancer Society; 2012.

19. Conselho Regional de Medicina do Estado de São Paulo. Cuidado Paliativo. São Paulo: Cremesp; 2008.

20. Parikh RB, Kirch RA, Smith TJ, Temel JS. Early specialty palliative care--translating data in oncology into practice. N Engl J Med. 2013;369(24):2347-51.

21. Menezes RA. Em busca da boa morte: antropologia dos cuidados paliativos. Rio de Janeiro: Fiocruz; 2004.

22. Bruera E, Yennurajalingam S. Palliative care in advanced cancer patients: how and when? Oncologist. 2012;17(2):267-73.

23. Academia Nacional de Cuidados Paliativos. Manual de cuidados paliativos. 2 ed. Rio de Janeiro: Diagraphic; 2009.

24. Mazanec P, Prince-Paul M. Integrating palliative care into active cancer treatment. Semin Oncol Nurs. 2014;30(4):203-11.

25. Floriani CA, Schramm FR. Desafios morais e operacionais da inclusão dos cuidados paliativos na rede de atenção básica. Cad Saúde Pública. 2007;23(9):2072-80. 


\section{Abstract}

Introduction: Advances and improvements in diagnosis and treatment of cancer, in Brazil and all over the world, have led to an increasing number of cancer survivors. While promising, the increasing in survivors results in more people living with chronic and delayed effects of cancer and its treatments, prompting a whole new reflection about the social impacts, formation of public policy and the models of care for the provision of palliative care in oncology. Objective: To understand the interdependent and complementary relationships between the application of the concept of palliation and quality of survival in the cancer patient from a multidisciplinary team perspective. Method: Qualitative research, based on the hermeneutic-dialectic approach, performed in an reference oncology hospital in Rio de Janeiro, attended by nine health care professional members of the multidisciplinary team, with data produced by two focus groups, between July and August 2015. Results: Four broad empirical categories were identified: notion of survival in oncology; survivorship; palliation and line care in oncology; palliation and quality of survivorship in oncology; and survivorship and evaluation of the institutional palliation model. Conclusion: The palliation needs of cancer survivors require specific cancer control policies which involve the formation of a palliation care planning model guided by a broader definition of survivorship based on studies of the physical, emotional, social and economic needs of this growing group.

Key words: Survival; Quality of Life; Palliative Care; Neoplasms; Qualitative Research.

\section{Resumen}

Introducción: Los avances y mejoras en el diagnóstico y tratamiento del cáncer han proporcionado, en Brasil y en el mundo, un aumento en el número de sobrevivientes. Aunque prometedor, el aumento de los sobrevivientes resulta en más personas conviviendo con los efectos crónicos y tardíos del cáncer y sus tratamientos, que requiere una nueva reflexión sobre los impactos sociales y la formación de las políticas públicas y de los modelos de atención para la prestación de cuidados paliativos en oncología. Objetivo: Comprender la relación de interdependencia y complementariedad entre la aplicación del concepto de cuidados paliativos y la calidad de la supervivencia de pacientes oncológicos, a la vista del equipo multidisciplinario. Método: Investigación cualitativa basada en la hermenéutica dialéctica, llevado a cabo en un hospital de referencia en oncología en Río de Janeiro, con la participación de nueve profesionales de la salud, miembros del equipo multidisciplinario, con los datos producidos en dos grupos focales, entre julio y agosto de 2015. Resultados: Se identificaron cuatro grandes categorías empíricas: noción de supervivencia en oncología; supervivencia, paliación y linea de cuidados en oncología; paliación y calidad de la supervivencia en oncología; y supervivencia y evaluación del modelo de la paliación institucional. Conclusión: Las necesidades de paliación de los sobrevivientes de cáncer requieren políticas de control de cáncer que incluyan la formación de un modelo de planeación de la atención, guiado por una definición más amplia de la supervivencia, basándose en los estudios de las necesidades físicas, emocionales, sociales y económicos de este creciente grupo.

Palabras clave: Supervivencia; Calidad de Vida; Cuidados Paliativos; Neoplasias; Investigación Cualitativa. 